PROCEEDINGS OF THE

AMERICAN MATHEMATICAL SOCIETY

Volume 127, Number 5, Pages 1567-1570

S 0002-9939(99)05274-0

Article electronically published on February 4, 1999

\title{
STRONGLY EXPOSED POINTS IN UNIFORM ALGEBRAS
}

\author{
PAUL BENEKER AND JAN WIEGERINCK
}

(Communicated by Dale Alspach)

\begin{abstract}
In this paper we show that the unit ball of an infinite dimensional function algebra has no strongly exposed points.
\end{abstract}

\section{IntRoduction}

In Banach spaces $X$ one often looks at the geometry of the unit ball, $\operatorname{Ball}(X)=$ $\{x \in X:\|x\| \leq 1\}$, where on the boundary one distinguishes between extreme and exposed points. A point $f$ in $\partial \operatorname{Ball}(X)$ is called exposed if there exists a continuous linear functional $L$ on $X$ such that $L(f)=\|L\|=1$ and $f$ is the only point in the unit ball that is mapped to 1 . In general it is not easy to see if $\partial \operatorname{Ball}(X)$ contains any extreme or exposed points, but in practical situations there are positive results. The famous Krein-Milman theorem, for example, asserts that in a dual Banach space the unit ball is the weak ${ }^{*}$ closed convex hull of its extreme points. (So $L^{1}$ is a dual space only in a few trivial cases.)

There is another refinement of the concept of exposedness: we call $f \in \partial \operatorname{Ball}(X)$ strongly exposed if there exists $L \in X^{*}$ with the properties $L(f)=\|L\|=1$ and for any sequence $\left(g_{n}\right)_{1}^{\infty}$ in $X$ such that $\lim _{n \rightarrow \infty}\left\|g_{n}\right\|=\lim _{n \rightarrow \infty} L\left(g_{n}\right)=1$, $\lim _{n \rightarrow \infty} g_{n}=f$ in $X$. Clearly a strongly exposed point is exposed.

In this paper we show that in any infinite dimensional uniform algebra (with identity) the unit ball does not have any strongly exposed points. Of course we can always interpret a uniform algebra as a closed subalgebra of the continuous functions on some compact (Hausdorff) space $K$, via the Gelfand transform, where it separates the points of $K$. This is the setting we wish to work in: a closed subalgebra $\mathcal{A}$ of $C(K)$ separating the points of $K$, containing the constants; in short, a function algebra (on $K$ ). Our result was inspired by looking at $H^{\infty}$ and the disc algebra first (where the proofs can be even more elementary/explicit, albeit slightly longer; for a description of the exposed points in these spaces; see [3], [1] and [2] respectively); the disc algebra (which plays a crucial role in our approach) is the uniform closure of the polynomials on the (closed) unit disc in $\mathbb{C}$. A trivial but important remark is that for $f$ in the (closed) subalgebra $\mathcal{A}$ of $C(K),\|f\| \leq 1$, and $g$ in the disc algebra, $g \circ f \in \mathcal{A}$.

Received by the editors April 20, 1998 and, in revised form, October 6, 1998.

1991 Mathematics Subject Classification. Primary 46E99; Secondary $46 J 10$.

Key words and phrases. Strongly exposed point, function algebra.

The research of the first author was supported by the Netherlands research organization NWO.

(C)1999 American Mathematical Society 
We state the results in the next section, together with the proofs. Along the way we also show how these results can be used to rule out duality of Banach spaces. Finally, we put the results into perspective.

\section{The RESUlts}

In our proof of the main result we will make use of the following two lemmas, the proofs of which will be outlined at the end of this section.

Lemma 1. Let $\Omega \subset \mathbb{C}$ be the interior of the Jordan curve $\{z \in \mathbb{C}:|z| \leq 1,|\arg z|=$ $\left.(1-|z|)^{2}\right\} \cup\{0\}$. Then

$$
\lim _{n \rightarrow \infty} \sup _{z \in \Omega}\left|1-z^{n}\right|=1 .
$$

In what follows $\phi: \Delta \rightarrow \Omega$ will be a fixed Riemann map of the unit disc to $\Omega-$ an element of the disc algebra - that maps 1 to 1 .

Lemma 2. If $F$ is strongly exposed in $\partial \operatorname{Ball}(\mathcal{A})$, then for all $x \in K,|F(x)|=1$.

We are now ready for the main result:

Main Theorem. The unit ball of an infinite dimensional function algebra $\mathcal{A} \subset$ $C(K)$ has no strongly exposed points.

Proof. Since $\mathcal{A}$ separates the points of $K, K$ is infinite and there must be infinitely many strong boundary points $t \in K$; i.e., given an arbitrary open neighborhood $V$ of $t$, we can find a function $f \in \mathcal{A}$ such that $f(t)=\|f\|=1$, while $|f|<1$ outside of $V$. (The supremum of $|f(t)|$ over all strong boundary points $t$ equals $\|f\|$ for any $f \in \mathcal{A}$, so there are infinitely many such $t$; see [5].)

Suppose $F \in \partial \operatorname{Ball}(\mathcal{A})$ is exposed w.r.t. the functional $L$. Using the HahnBanach theorem it is easy to see that $L$ must be of the form

$$
L: g \in \mathcal{A} \mapsto \int_{K} g \bar{F} d \mu,
$$

for a certain probability measure $\mu$ (that must be supported on the set where $|F|=1)$. We can find a sequence of open sets $\left(V_{n}\right)_{1}^{\infty}$, each containing a strong boundary point $k_{n}$ with corresponding $f_{n} \in \mathcal{A}$, such that

$$
\sum_{n=1}^{\infty} \mu\left(V_{n}\right)<\infty
$$

But then the functions $1-\left(\phi \circ f_{n}\right)^{n} \in \mathcal{A}$ tend to 1 boundedly and pointwise outside of $\bigcap_{k=1}^{\infty} \bigcup_{n=k}^{\infty} V_{n}$, a set of $\mu$-measure 0 , as $n \rightarrow \infty$. An easy application of the dominated convergence theorem yields that $L\left(\left(1-\left(\phi \circ f_{n}\right)^{n}\right) F\right) \rightarrow 1$, whereas $\left\|\left(1-\left(\phi \circ f_{n}\right)^{n}\right) F\right\| \rightarrow 1$, yet

$$
\left\|\left(1-\left(\phi \circ f_{n}\right)^{n}\right) F-F\right\|=\left\|\left(\phi \circ f_{n}\right)^{n} F\right\| \geq\left|F\left(k_{n}\right)\right|,
$$

so that by Lemma 2, $F$ cannot be strongly exposed.

Proof of Lemma 1. By the maximum modulus theorem we need to show that

$$
\lim _{n \rightarrow \infty} \sup _{z \in \partial \Omega}\left|1-z^{n}\right|=1 .
$$

For $z \in \partial \Omega$ :

$$
\left|1-z^{n}\right|^{2}=1+|z|^{2 n}-2|z|^{n} \cos \left(n(1-|z|)^{2}\right) .
$$


If $n(1-|z|)^{2} \leq \frac{1}{\sqrt{n}}$, then the cosine is at least $\frac{1}{2}$ and $\left|1-z^{n}\right|^{2} \leq 1$. On the other hand, if $n(1-|z|)^{2} \geq \frac{1}{\sqrt{n}}$, then $|z|^{n}$ is bounded above by $\left(1-\frac{1}{n^{3 / 4}}\right)^{n}$ which behaves like $e^{-n^{1 / 4}}$.

Proof of Lemma 2. Take $e^{i \theta} \in \mathbb{T}$. $E_{\theta}:=\left\{x \in K: F(x)=e^{i \theta}\right\}$. The function $G=G_{\theta}=\phi \circ\left(e^{-i \theta} F\right)$ in $\mathcal{A}$ peaks on $E_{\theta}$, and is such that $\limsup _{n \rightarrow \infty}\left\|1-G^{n}\right\| \leq 1$, and $1-G^{n} \rightarrow 1$ boundedly and pointwise outside of $E_{\theta}$. As in the proof of the theorem it follows that $\mu\left(E_{\theta}\right) \neq 0$ if $E_{\theta} \neq \emptyset$. Hence $E=F\left(F^{-1}(\mathbb{T})\right)$ is a countable compact subset of $\mathbb{T}$, thus a peak set for a function $\psi$ in the disc algebra. But then $(\psi \circ F) F$ and $F$ coincide on the support of $\mu$ showing that $(\psi \circ F) F=F$, and thus $F(x)=0$ or $F(x) \in E$.

Now if $E$ were infinite, it would contain infinitely many isolated points $e^{i \theta_{n}}$, along which $\mu\left(E_{\theta_{n}}\right) \rightarrow 0$. Take a function $\psi_{n}$ in the disc algebra that peaks on $E \backslash E_{\theta_{n}}$, such that $\left|\psi_{n}\left(e^{i \theta_{n}}\right)\right| \leq \frac{1}{2}$. It is easy to check that $L\left(\left(\psi_{n} \circ F\right) F\right) \rightarrow 1$, while $\left\|\left(\psi_{n} \circ F\right) F-F\right\| \geq \frac{1}{2}$ contradicting the assumption that $F$ is strongly exposed. Hence $E$ is a finite set, say $E=\left\{e^{i \theta_{1}}, \ldots, e^{i \theta_{N}}\right\}$. Using standard arguments it follows that $1_{E_{\theta_{1}}}, \ldots, 1_{E_{\theta_{N}}} \in \mathcal{A}$. Also, $1 \in \mathcal{A}$ so that $1_{F^{-1}(0)} \in \mathcal{A}$. Now the exposing functional $L$ cannot distinguish between the elements $F$ and $F+1_{F^{-1}(0)}$ of $\partial$ Ball, so that in fact $F$ is nowhere zero. We conclude that $F$ only assumes the values $e^{i \theta_{1}}, \ldots, e^{i \theta_{N}}$ on $K$; in particular, for all $x \in K,|F(x)|=1$.

Corollary. No (infinite dimensional) separable function algebra is a dual space.

One way of proving this result is by using the fact that in a separable dual space the unit ball is the closed convex hull of its strongly exposed points; see [6]. For example, the disc algebra is not a dual space. We thank the referee for pointing out that the corollary is also a consequence of the fact that the space $c_{0}$ of sequences on $\mathbb{N}$ that tend to zero, can be isometrically embedded into each separable function algebra (use the generalized Rudin-Carleson theorem, pg. 191 in [4] with a suitably chosen compact set $F$ ), combined with the absence of extreme points in the unit ball of $c_{0}$.

Looking back, we see that for function algebras, the condition $L\left(g_{n}\right) \rightarrow 1$ in the definition of a strongly exposed point $f$ translates to convergence of the functions $g_{n}$ to $f$ with respect to a single measure, which is of course for $C(K)$ not as strong as the desired uniform convergence (unless $K$ is pathological). A simple proof using Urysohn's lemma uses $C(K)$ 's algebraic structure only in a minimal way. Couple that with the fact that any Banach space is linearly and isometrically embeddable as a (closed) subspace of some $C(K)$, and it is no longer obvious why strongly exposed points should not exist for general subalgebras of $C(K)$.

\section{REFERENCES}

[1] R.R. Phelps, Extreme points in function algebras, Duke Math. J. 32 (1965), 267-277. MR 31:3890

[2] S. Fisher, Exposed points in spaces of bounded analytic functions, Duke Math. J. 36 (1969), 479-484. MR 41:4247

[3] E. Amar and A. Lederer, Points exposés de la boule unité de $H^{\infty}(D)$, C.R. Acad. Sci. Paris Sér. A-B 272 (1971), A449-A452. MR 44:788

[4] G.M. Leibowitz, Lectures on Complex Functions, Scott, Foresman and Company (1970). MR 55:1072 
[5] E.L. Stout, The theory of uniform algebras, Bogden \& Quigley (1971). MR 54:11066

[6] R.R. Phelps, Dentability and extreme points in Banach spaces, J. Funct. Anal. 17 (1974), 78-90. MR 50:5427

Faculty of Mathematics, Computer Science, Physics and Astronomy (Wins), UniverSity of Amsterdam, Plantage Muidergracht 24, 1018 TV Amsterdam, The Netherlands

E-mail address: beneker@wins.uva.nl

E-mail address: janwieg@wins.uva.nl 